\title{
A Novel Method for Fast Processing of Large Remote Sensed Image
}

\author{
Adriano Mancini ${ }^{1}$, Anna Nora Tassetti ${ }^{2}$, Alessandro Cinnirella ${ }^{3}$, \\ Emanuele Frontoni ${ }^{1}$, and Primo Zingaretti ${ }^{1}$ \\ 1 Dipartimento di Ingegneria dell'Informazione, \\ Università Politecnica delle Marche Ancona, Italy \\ 2 Dipartimento di Ingegneria Civile, Edile e Architettura, \\ Università Politecnica delle Marche Ancona, Italy \\ 3 Dipartimento di Scienze Geologiche, Università degli Studi Roma Tre \\ \{mancini, frontoni, zinga\}@dii. univpm.it, \\ n.tassetti@univpm.it, \\ a.cinnirella@uniroma3.it
}

\begin{abstract}
In this paper we present a novel approach to reduce the computational load of a CFAR detector. The proposed approach is based on the use of integral images to directly manage the presence of masked pixels or invalid data and reduce the computational time. The approach goes through the challenging problem of ship detection from remote sensed data. The capability of fast image processing allows to monitor the marine traffic and identify possible threats. The approach allows to significantly boost the performance up to 50x working with very high resolution image and large kernels.
\end{abstract}

Keywords: Remote Sensing, CFAR, VHR imagery, SAR, ship detection, integral image.

\section{Introduction}

Nowadays near real time imagery services has become a useful reality in disaster and crisis-management. For example, it played a key role in the management of the Haiti earthquake in 2010. In that case and during critical events in general, the analysis of remote sensed images provides useful information to the crisis management cycle in all its phases: from assisting urban risk reduction measures to smart identification of damages after a calamity, planning recovery and reconstruction activities. The main problem is the time required to process each image. The availability of very high resolution images imposes new constraints and challenges for processing algorithms.

In this paper we focus on the problem of object extraction from high resolution remote sensed data (optical and SAR images). The main innovation is the capability of fast statistics computation for ship extraction also in presence of masked areas. The well-known approach of Integral Image, as proposed by Viola and Jones [16], is used to solve the problem of rapid object detection. 
The aim of the paper is to show how is possible to speed-up the processing, especially working with large kernels, and keep on using common algorithms as the CFAR. We introduce a novel application of Integral Image to fast extract objects as boat/ship. The boat/ship detection in remote sensing is a challenging point to solve many problems as (marine) border surveillance and marine traffic monitoring. An interesting review can be found here [17] where some important application fields and their main requirements for a vessel detection system are summarized. DECLIMS (Detection, Classification and Identification of Marine Traffic from Space) [1] was an interesting EU project with the overall objective "to provide a focus for research into the use of satellite imagery for maritime vessel detection, classification and identification". Dealing with ship detection, the main problem is the required time to process an image. The availability of very high resolution imagery allows to detect small ships and reduce the false positive rate occurring with long ships. Many approaches have been proposed for both optical and SAR data-set. Optical images have advantages over SAR data: high spatial resolution (it is possible to obtain images with a pixel size of $0.5 \mathrm{~m}$ ) and availability of a multi-spectral platform. On the contrary their main drawback (advantage of SAR) is that they are not available during bad weather conditions (clouds, night).

Dealing with optical images, many approaches are based on image segmentation 418, especially by automatic thresholding (e.g. histogram-based, local, spatial) [5]. The segmentation is necessary to separate possible candidates as ships, clouds, islands, waves and marine platforms. The following processing phases, with the aim of identifying ships, are mainly based on shape and texture analysis 9. About SAR images and their state of art a good review can be found in [6]. The problem of SAR images is the presence of speckle noise, boosting the probability of false positives. The noise in SAR images must be reduced to increase the accuracy of detection. Many filters based on minimum mean square error (MMSE) theory has been proposed as Lee/, Enhanced Lee [12 13], Kuan [1], Frost/Enhanced Frost [8]. An evolved method to isolate possible candidates is represented by the Constant False Alarm Rate (CFAR) methodology. The CFAR has been widely used in the context of ship detection [103. In particular, an open-source software for SAR processing, the Next ESA SAR Toolbox (NEST), has been developed for reading, post-processing, analysing and visualising the large data archive. NEST supports handling of products from third party missions including JERS-1, ALOS PALSAR, TerraSAR-X, Radarsat-2 and Cosmo-Skymed 7/2]. NEST has a dedicate object extraction module to detect ship from SAR images. The main limit of this module, based on CFAR, is the long time required to process an image in the case of high resolution and medium-size ships $(<250 \mathrm{~m})$. In this paper we present an approach to boost the performance of a CFAR detector. The approach is based on integral images [14], and so taking into account also the presence of not valid data and/or masked data. Not valid data and/or masked data represent pixels that should be overlooked by the CFAR. The approach here proposed is partially independent by the clutter probability density function which can be Gaussian or K-Gamma [15. 
The advantages of the proposed algorithm are particularly evident when large windows ( $>100$ pixels) have to be considered to detect objects.

The paper is organized as follows. In Section 2 the new approach and the processing methodology is presented. In Section 3 experimental results of ship extraction from optical and SAR images are discussed. Section 4 outlines conclusions and future works.

\section{Methodology}

In this section the ship detection methodology is discussed: working with remote sensed data it is shown how is possible to boost the performance of CFAR approach. To simplify matters, it is assumed that the sea clutter is described by a Gaussian probability density function.

\subsection{Proposed Approach}

The adaptive thresholding, as depicted in Fig. 11, considers three different areas:

- Target area;

- Guard area;

- Background area.

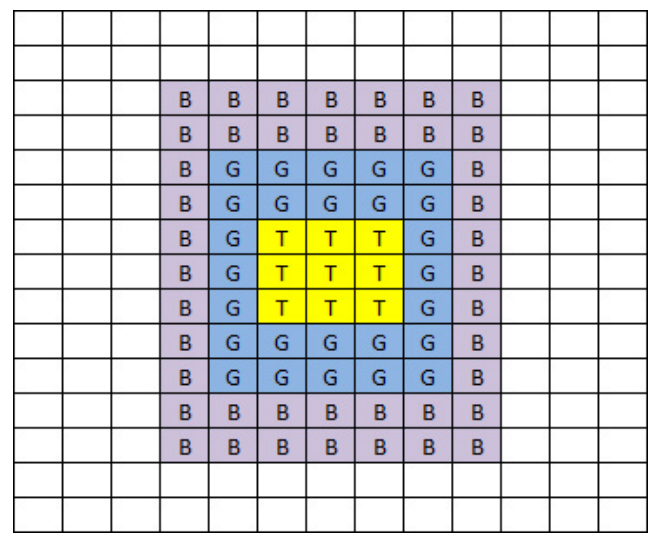

Fig. 1. Example of a window in adaptive thresholding

The basic idea is to search pixels unusually bright when compared to the surrounding pixels. Let $x_{t}$ be the pixel under test and $T$ be a given threshold, then the detection criterion can be expressed as:

$$
x_{t}>T \Leftrightarrow x_{t} \text { is a target }
$$


When the target window contains more than one pixel, the operator uses the following detection criterion:

$$
\mu_{t}>T \Leftrightarrow x_{t} \text { is a target }
$$

where $\mu_{t}$ is the mean value of pixels in the target window. The size of the target window should be set to the minimum ship size to detect. In the Gaussian case the Eq. 2 is modified in the following way:

$$
\mu_{t}>\mu_{b}+\sigma_{b} \cdot t
$$

where $\mu_{b}$ and $\sigma_{b}$ are respectively the mean value and the standard deviation of pixels in the background window. The $t$ value is chosen according to the desired Probability of False Alarm (PFA). The guard window is necessary to mask the target by the rest of noisy pixels. For this reason the size of this window should be set to the size of the largest target to detect. The size of the background window should be large enough to ensure an accurate calculation of the background statistics (in this case Gaussian). It is also necessary to consider that it is necessary to mask the presence of land and/or islands in order to avoid false positives and detect ships (Fig. 2).

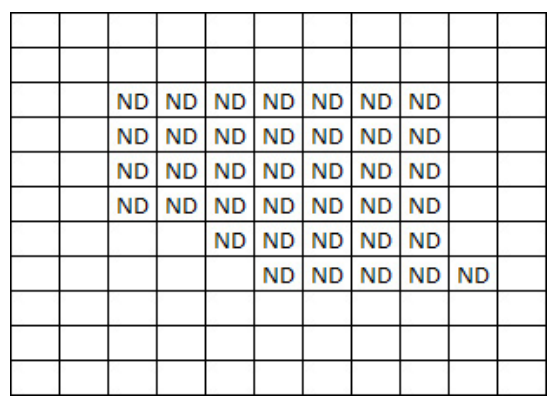

Fig. 2. Image with no data (ND) value for various pixels to mask the land

The ND value must be taken into account during the calculation of the statistics to avoid invalid results. If the image has a pixel size of $5 \mathrm{~m} \times 5 \mathrm{~m}$ and the largest object has a size of $250 \mathrm{~m}$, the size of the guard window must be large (e.g., kernel size 51x51). Moreover the size of background windows should consider an area of $600 \mathrm{~m} \times 600 \mathrm{~m}$ that corresponds to a kernel of $121 \times 121$. In this case it is obvious that very high resolution images require large kernels to detect ships. Large kernels cause long processing time if the kernels are not separable. The detection of a ship requires to evaluate the terms in the Eq. 3. For this reason it is necessary to calculate efficiently the terms $\mu_{t}, \mu_{b}$ and $\sigma_{b}$.

A first way to compute $\mu_{t}$ and $\mu_{b}$ is by means of classical separable kernels to calculate the average value on a given size window. The kernel $(\mathrm{N} \times \mathrm{N})$ is structured as follows: 


$$
\left[\begin{array}{ccc}
\frac{1}{N} & \cdots & \frac{1}{N} \\
& \ddots & \\
\frac{1}{N} & & \frac{1}{N}
\end{array}\right]=\frac{1}{N}[\overbrace{1 \cdots 1}^{N}]^{T}[\overbrace{1 \cdots 1}^{N}]
$$

From Eq. 4 it is possible to observe that the complexity to calculate the average of a set of pixels around a given pixel, considering a kernel size of $\mathrm{N}$ x $\mathrm{N}$, is $O(N)$ instead of $O\left(N^{2}\right)$ if the separable kernel solution is adopted. To obtain the value of $\mu_{b}$ it is necessary to calculate $\mu_{t}$ and $\mu_{g}$ where $\mu_{g}$ represents the mean value of pixels in the guard window. The kernel size for target, guard and background are $t \cdot t, g \cdot g$ and $b \cdot b$. There are two options to calculate $\mu_{b}$. The first one is to consider a not-separable kernel. This choice is not the best one due to the size of background windows that is usually large considering that $t<g<b$. The second option is to calculate $\mu_{b}$ in the following way:

$$
\tilde{\mu}_{b}=\frac{b \cdot \mu_{b}-g \cdot \mu_{g}}{b-g}
$$

where $\mu_{b}$ is calculated using a separable kernel and $\tilde{\mu}_{b}$ is the final value not considering the guard window. About the standard deviation:

$$
\begin{aligned}
& \sigma_{b}^{2}=E\left[x_{b}^{2}\right]-E\left[x_{b}\right]^{2} \\
& \sigma_{b}^{2}=E\left[x_{b}^{2}\right]-\left(\tilde{\mu}_{b}\right)^{2} \\
& E\left[x_{b}^{2}\right]=\frac{b \cdot \bar{\mu}_{b}-g \cdot \bar{\mu}_{g}}{b-g} \\
& \hat{\mu}_{b}=E\left[x_{b}^{2}\right] \\
& \sigma_{b}^{2}=\hat{\mu}_{b}-\left(\tilde{\mu}_{b}\right)^{2}
\end{aligned}
$$

where $x_{b}^{2}$ represents the squared value of pixels in the background window, $\tilde{\mu}_{b}$ is derived from Eq. 6. $\bar{\mu}_{b}$ and $\bar{\mu}_{g}$ are the mean value of "squared value" pixels, respectively of background and guard window, using separable kernels. For example to calculate the $\tilde{\mu}_{g}$ it is necessary to:

- Calculate $\mu_{g}$;

- Calculate $\mu_{b}$;

- Apply the Eq. 5 .

The critical point is that if $b$ is high then two runs of separable kernels are necessary: this aspect is critical especially for large kernels. An interesting time saving alternative is to use the concept of Integral Image also known as Summed Area Table (SAT). The integral image (see Eq. 7) can be used to efficiently generate the sum of values in a rectangular region of interest of a given image.

$$
I(x, y)=\sum_{i=0}^{x} \sum_{j=0}^{y} i m g(i, j)
$$

However the integral image can be easily computed in a single pass (Eq. 7):

$$
I(x, y)=i m g(x, y)+I(x-1, y)+I(x, y-1)+I(x-1, y-1)
$$


The great advantage of using an integral image is the capability to calculate the sum of all pixel values in a given rectangular Region Of Interest (ROI) using the following expression:

$$
S_{i m g}(\hat{x}, \hat{y})=\sum_{A_{x} \leq \hat{x} \leq C_{x}} \sum_{A_{y} \leq \hat{y} \leq C_{y}} i m g(\hat{x}, \hat{y})=I(C)+I(A)-I(B)-I(D)
$$

where $A, B, C$ and $D$ represent the four points delimitating the ROI. The calculation of $\mu_{g}$ and $\mu_{b}$ using the Eq. 9 is trivial. Eighth basic operations allows to calculate the mean value of pixels in a given ROI. The great advantage of the integral image in this context is the capability to model also the presence of no-data, mainly caused by the necessity to mask the land. No-data pixels can also represent invalid data from the instrument installed on-board of the satellite. The no-data pixels can significantly influence the statistics and then the detection and so, to be rigorous, they have to be considered when statistics are calculated. We take into account this aspect by calculating a new integral image on the mask image. The mask image $M$ has the same size of the original image with 0 for data to be considered and 1 for no-data (invalid data or masked by the presence of land). The Eq. 5 is modified in the following way:

$$
\begin{aligned}
\tilde{\mu}_{b} & =\frac{\left(b-n d_{b}\right) \cdot \mu_{b}-\left(g-n d_{g}\right) \cdot \mu_{g}}{b-g-n d_{b}} \text { with } n d_{b}=S_{M}(\hat{x}, \hat{y}) \\
A & =\left(\hat{x}-\frac{b}{2}, \hat{y}-\frac{b}{2}\right) B=\left(x+\frac{b}{2}, \hat{y}-\frac{b}{2}\right) \\
C & =\left(x+\frac{b}{2}, \hat{y}+\frac{b}{2}\right) D=\left(x-\frac{b}{2}, \hat{y}+\frac{b}{2}\right)
\end{aligned}
$$

where $n d_{b}$ is the number of no-data pixels inside the given region of size $b \cdot b$; this value is derived from the integral image on the image $M$ representing the mask for land and invalid instrument data. $n d_{g}$ represents the number of no-data pixels inside the given region of size $g \cdot g$.

Using Eq. 10 it is possible to calculate the correct value of $\tilde{\mu}_{b}$ taking into account also the no-data value. In the same way the Eq. 6 can be modified to take into account the no-data pixels. The separable kernel is not able to manage this situation and a not separable kernel should be used. The main problem is the complexity roughly estimable as $O\left(N^{2}\right)$ with $N=b$. If $N$ is large the required time can be considerable. Using the proposed approach the complexity is $O(N)$ with a significant saving in terms of time. The approach here proposed is suitable for a Gaussian PDF but the methodology based on integral images can be easily extended also for other PDFs as K or Alpha-stable.

\subsection{Numerical Aspects}

Dealing with integral images it is important to consider the data type of original image and of integral one. The uint32 should be not sufficient to store the values of integral images especially when the standard deviation has to be calculated. The sum of the squared values of pixels could easily cause an overflow. For this reason in the case of large images it is necessary to use the uint64 data type. If uint64 is not sufficient it is necessary to modify the approach introducing 
the well known concept of image tiling. Usually a tile represents a sub-image that is easier to manage (from the point of view of memory and data type). The proposed approach of using integral images can be also used in a tiled approach. The size of a tile of a given image of size $\mathrm{W} \times \mathrm{H}$ is chosen according to the original image data type (usually uint8/int8 or uint16/int16). When using the tile it is important to describe how the tiles are generated from the original image. The size of a tile should be $W / N_{\text {tiles }} \cdot H / N_{\text {tiles }}$ but to ensure the continuity among contiguous tiles it is necessary to modify the position of a tile that should overlap in area; this aspect is depicted in Fig. 3 where $H_{T}$ is the height of a given tile.

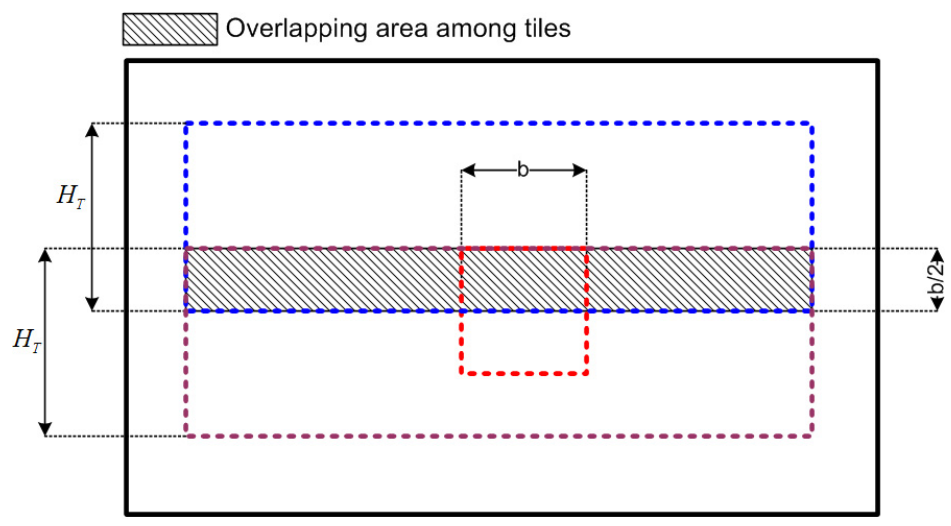

Fig. 3. Management of tiles

\section{Results}

In this section we present some results of image processing to detect ships in SAR images acquired by COSMO-SkyMed satellite. In Fig. 4 the test area (Southern Italy) and an example of land mask is shown. The image is a $D G M_{B}$ product (Detected Ground Multilook) with ascending orbit. The size is 4837 columns by 4081 rows. The pixel size is about $30 \mathrm{~m} \times 30 \mathrm{~m}$. The CFAR parameters are listed in Table 1. In Fig. [5 an example of ship detection is shown.

Table 1. List of parameters for ship detection

\begin{tabular}{lc}
\hline Parameter & Value \\
\hline Target window size & $80 \mathrm{~m}$ \\
Guard window size & $400 \mathrm{~m}$ \\
Background window size & $1000 \mathrm{~m}$ \\
PFA & $10^{-6.5}$ \\
\hline
\end{tabular}



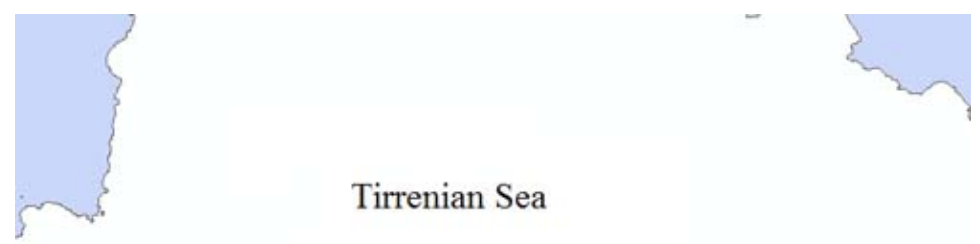

\section{Tirrenian Sea}

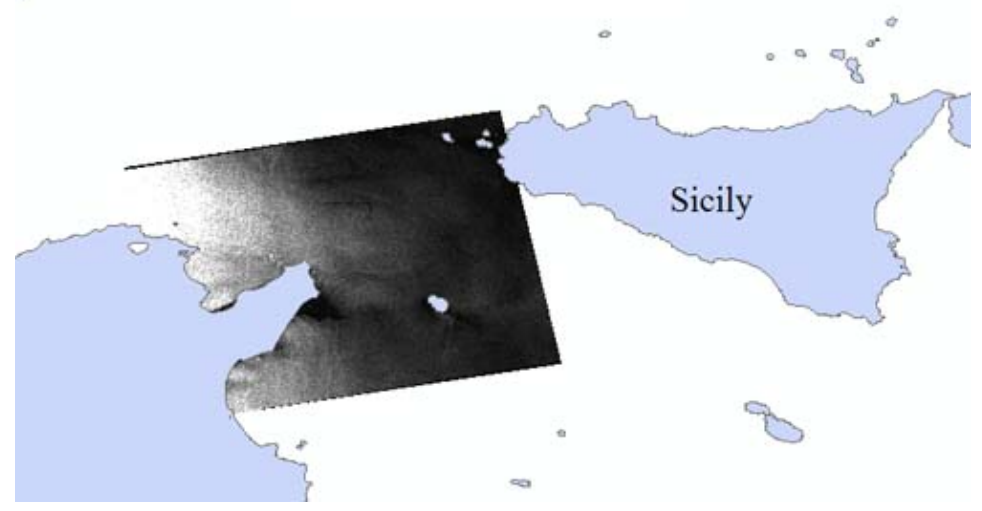

Fig. 4. Left: Test area. Right: Land mask overlaid with stretched SAR amplitude data

Table 2. Comparison of required time to process the data-set (just the detection phase; the image loading is not considered)

\begin{tabular}{llc}
\hline Approach & \multicolumn{2}{c}{ Required Time [s] Boost } \\
\hline Not-separable kernel & $106.0 \mathrm{~s}$ & $1 \mathrm{x}$ \\
Separable kernel & $10.5 \mathrm{~s}$ & $10 \mathrm{x}$ \\
Integral Image (our approach) & $5.0 \mathrm{~s}$ & $20 \mathrm{x}$ \\
\hline
\end{tabular}

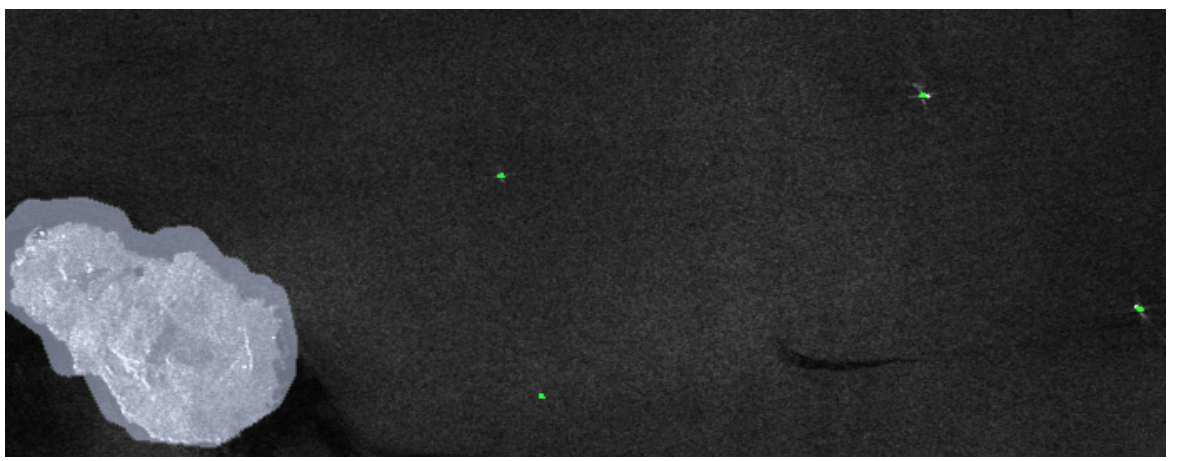

Fig. 5. Example of ship detection using CFAR detector; green pixels represent the ships 
In Table 2 the comparison of the required time to process the image is shown. It is important to underline how the integral image approach allows to manage no-data. The gain of performance is more obvious when the image has a lower pixel size (e.g. $3 \mathrm{~m} \times 3 \mathrm{~m}$ ) as available for some SAR product (COSMOSkyMed). Tests are carried out with this pixel size and show how the integral image approach meaningfully boosts the performance of the separable kernel one. A boost of $50 \mathrm{x}$ and more can be achieved with large kernels and very high resolution images.

\section{Conclusion and Future Works}

In this paper we presented a novel approach to work with a CFAR detector and improve it in terms of computational load and processing time. Integral images are used with an advantage in reducing the computational time and directly managing the presence of masked pixels (e.g., presence of land, invalid instrument data). The approach can be also extended to other kind of probability density function (e.g. K) and is useful especially working with very high resolution images where kernel and image sizes play a key role. The methodology has been successfully applied to face ship extraction from remote sensed data. The accuracy and precision of object extraction can be improved correlating the position of extracted objects with Automatic Identification System (AIS) data.

On going research plans to test the approach with an accelerated version of the proposed algorithm using a Compute Unified Device Architecture (CUDA) on a GPU and compare its performance with a CPU based approach.

Acknowledgements. The authors would like to thank the private company GMSpazio S.r.l for their support and the provided data-set. The suggestions of Eva Savina Malinverni at DICEA - UNIVPM have been really important.

\section{References}

1. Declims eu project website, https://declims.jrc.ec.europa.eu/home

2. Next esa sar toolbox - array, http://nest.array.ca/web/nest

3. Wackerman, C., et al.: Toward an automated ship and wake detection system (2006)

4. Barale, V., Gade, M.: Remote Sensing of the European Seas. Springer Science Business Media B.V. (2008), http://books .google.fr/books?id=9B3D5-HBTzkC

5. Corbane, C., Najman, L., Pecoul, E., Demagistri, L., Petit, M.: A complete processing chain for ship detection using optical satellite imagery. Int. J. Remote Sens. 31(22), 5837-5854 (2010), http://dx.doi.org/10.1080/01431161.2010.512310

6. Crisp, D., Science, D., Laboratory, T.O.A.I.S.: The State-of-the-art in Ship Detection in Synthetic Aperture Radar Imagery. Research report (Defence Science and Technology Organisation (Australia). DSTO Information Sciences Laboratory (2004), http://books.google.it/books?id=cdGvtwAACAAJ 
7. Engdahl, M., Minchella, A., Marinkovic, P., Veci, L., Lu, J.: Nest: An esa open source toolbox for scientific exploitation of sar data. In: 2012 IEEE International Geoscience and Remote Sensing Symposium (IGARSS), pp. 5322-5324 (2012)

8. Frost, V.S., Stiles, J.A., Shanmugan, K., Holtzman, J.: A model for radar images and its application to adaptive digital filtering of multiplicative noise. Pattern Analysis and Machine Intelligence, IEEE Transactions on PAMI-4(2), 157-166 (1982)

9. Huang, G., Wang, Y., Zhang, Y., Tian, Y.: Ship detection using texture statistics from optical satellite images. In: 2011 International Conference on Digital Image Computing Techniques and Applications (DICTA), pp. 507-512 (2011)

10. Huang, W., Chen, P., Yang, J., Fu, B., Xiao, Q., Yao, L., Zhou, C.: An improved cfar model for ship detection in sar imagery. In: Proceedings of the 2004 IEEE International Geoscience and Remote Sensing Symposium, IGARSS 2004, vol. 7, pp. 4719-4722 (2004)

11. Kuan, D.T., Sawchuk, A., Strand, T.C., Chavel, P.: Adaptive restoration of images with speckle. IEEE Transactions on Acoustics, Speech and Signal Processing 35(3), 373-383 (1987)

12. Lee, J.S.: Speckle suppression and analysis for synthetic aperture radar images. Optical Engineering 25(5), 255636-255636 (1986)

13. Lopes, A., Nezry, E., Touzi, R., Laur, H.: Maximum a posteriori speckle filtering and first order texture models in sar images. In: 10th Annual International Geoscience and Remote Sensing Symposium, IGARSS 1990. 'Remote Sensing Science for the Nineties', pp. 2409-2412 (1990)

14. Shafait, F., Keysers, D., Breuel, T.M.: Efficient implementation of local adaptive thresholding techniques using integral images, 681510-681510-6 (2008)

15. Tunaley, J.: Ship detection in sar imagery. Tech. rep., LRDC Technical Report (December 2010)

16. Viola, P., Jones, M.: Rapid object detection using a boosted cascade of simple features. In: Proceedings of the 2001 IEEE Computer Society Conference on Computer Vision and Pattern Recognition, CVPR 2001, vol. 1, pp. I-511-I-518 (2001)

17. Willhauck, G., et al.: Object-oriented ship detection from vhr satellite images. Tech. rep. (2005)

18. Zhu, C., Zhou, H., Wang, R., Guo, J.: A novel hierarchical method of ship detection from spaceborne optical image based on shape and texture features. IEEE Transactions on Geoscience and Remote Sensing 48(9), 3446-3456 (2010) 